\title{
Correspondence
}

\section{Patients who experience a perioperative anaphylactic reaction should not be skin-tested too early}

To the Editor:

We read with interest the article by Cummings et al. ${ }^{1}$ concerning the report of a perioperative anaphylactic reaction ascribed to fentanyl. As the authors rightly suggested, this case report clinically evoked an immediate allergic hypersensitivity reaction ${ }^{2}$ according to the time onset of the reaction and likewise the clinical symptoms in favour of a grade III reaction. ${ }^{3}$ As noted by the authors, opioid-induced anaphylaxis is rare. In this patient, positive skin-test reactions were evidenced to both fentanyl and succinylcholine. Given that the patient did not receive succinylcholine, a judicious conclusion was that fentanyl seemed the likely cause. Nevertheless, in that case report, an anaphylaxis to rocuronium cannot be definitely ruled out. If skin tests (prick-test and/or intradermal tests) remain the gold standard for the detection of IgE-dependent allergies, ${ }^{4}$ these skin tests should, whenever possible, be performed after a delay of at least four to six weeks according to current guidelines. ${ }^{5-7}$ This delay is necessary in order to avoid false negative reactions due to mast cell depletion occurring after an anaphylactic reaction. When performed earlier (within one week after the event in the case reported): i) only positive results can be taken into account; and ii) skin tests should be repeated after the sixth postoperative week. Within that time, and while this second assessment is still pending, rocuronium and succinylcholine should not be re-injected.

Furthermore, the discordance between the conclusions of the allergologist and the anesthesiologist is troublesome. The former suggested avoiding "fentanyl and all paralytic agents if possible", whereas the anesthesiologist team informed the patient that "she could receive neuromuscular blocking drugs (NMBDs) in the future if circumstances warranted" Such a distorsion in the conclusion of an allergological assessment following a perioperative reaction should be avoided. One of the most important goals of the allergological assessment performed after a perioperative anaphylactic reaction, in addition to identifying the culprit agent, is to propose, based on the nega- tive skin tested NMBDs, those which could be safely administered for future procedures. Indeed, when a prick-test or/and an intradermal test with a NMBDs is/are positive, investigation for cross-reactivity with other commercialized NMBDs must be performed by the intradermal route, taking into account the maximal concentration of the drug that must not be exceeded. ${ }^{7}$ These NMBDs should therefore be clearly identified and proposed for future procedures according to a consensus between the anesthesiologist and the allergologist. Such cooperation is mandatory in order to provide a non-ambiguous response essential for the future safety of the patient.

Pascale Dewachter MD PhD

Claudie Mouton-Faivre MD

Hôpital Central, Centre Hospitalier Universitaire, Nancy, France

E-mail: pascale.dewachter@yahoo.fr

Accepted for publication May 22, 2007.

\section{References}

1 Cummings KC $3^{\text {rd }}$, Arnaut K. Case report: Fentanylassociated intraoperative anaphylaxis with pulmonary edema. Can J Anesth 2007; 54: 301-6.

2 Johansson SG, Houribane JO, Bousquet J, et al; EAACI (the European Academy of Allergology and Clinical Immunology) nomenclature task force. A revised nomenclature for allergy. An EAACI position statement from the EAACI nomenclature task force. Allergy 2001; 56: 813-24.

3 Ring J, Messmer K. Incidence and severity of anaphylactoid reactions to colloid volume substitutes. Lancet 1977; 1: 466-9.

4 Anonymous. The use of in vitro tests for $\operatorname{IgE}$ antibody in the specific diagnosis of IgE-mediated disorders and in the formulation of allergen immunotherapy. American Academy of Allergy and Immunology. J Allergy Clin Immunol 1992; 90: 263-7.

5 The Association of Anaesthetists of Great Britain and Ireland and British Society for Allergy and Clinical Immunology. Suspected anaphylactic reactions associated with anaesthesia. 2003 August. Available from URL; http://www.aagbi.org/publications/guidelines/ docs/anaphylaxis03.pdf (accessed April 18, 2007).

6 The French Society of Anesthesiology and Intensive Care Medicine. Clinical practice guidelines. Reducing the 
risk of anaphylaxis during anaesthesia. Abbreviated text. Ann Fr Anesth Reanim 2002; 21 Suppl 1: 7-23.

7 Joint Task Force on Practice Parameters; American Academy of Allergy, Asthma and Immunology; American College of Allergy, Asthma and Immunology; Joint Council of Allergy, Asthma and Immunology. The diagnosis and management of anaphylaxis: an updated practice parameter. J Allergy Clin Immunol 2005; 115(3 Suppl 2): S483-523.

\section{Reply:}

I thank Drs. Dewachter and Mouton-Faivre for their thoughtful comments on our case report. I believe, however, that certain points require comment and clarification.

First, they state that a reaction to rocuronium cannot be ruled out because of the brief time between the event and the subsequent skin testing. We agree that published recommendations ${ }^{1}$ suggest a delay of four to six weeks from the event to allow regeneration of reactive IgE and mast cells. Due to logistical issues, this was not feasible in our situation. The patient did, however, react to succinylcholine, which, if their reasoning is true, would require antibodies to two separate neuromuscular blocking drugs (NMBDs) instead of one. This is certainly possible, and we subsequently referred the patient for repeat skin testing. Fentanyl allergy, however, is exceedingly rare, with only case reports documenting occurrences. Our bypothesis of causation by fentanyl rests partially on the fact that a false positive skin test to fentanyl should be considerably less likely than a NMBD. The work by Dhonneur et al. ${ }^{2}$ highlights the hazards of interpreting a positive skin test for rocuronium or vecuronium.

The results of the patient's repeat skin testing were quite unexpected: her only reaction was to the positive control (histamine), with no reaction to any of the NMBDs or fentanyl. The patient's allergist believes that these results represent a false negative, and that she has a low level of antibodies to one of the drugs tested. This situation has been encountered previously with Hymenoptera venom allergy and is a vexing problem in the allergy literature. ${ }^{3}$ The best advice we can offer the patient at this point is to avoid fentanyl, succinylcholine, and rocuronium. If she were to require surgery with paralysis in the future, cisatracurium may be the wisest choice. One cannot, however, be entirely certain, as falsenegative results have also been reported for NMBDs. ${ }^{4}$

Finally, I would like to comment on the assertion that we distorted the conclusions of the allergist. Due to the ambiguity of the original test results, the allergist drew a rational conclusion: avoid all paralytic agents. We bave made the patient aware of the uncertainty from the skin tests. Our position, however, is that this could pose an unnecessary hazard to the patient in case of future surgery. General anesthesia with airway management, particularly in an emergency, could be much more hazardous without the use of a NMBD. The patient's lack of response (twice) to cisatracurium is somewhat reassuring, as she did not receive this drug prior to her initial reaction. I apologize for the lack of clarity on this point in the case report. A wiser phrasing might have been: "It is likely that the patient may receive selected NMBDs, such as cisatracurium, in the future if circumstances warranted.”

Kenneth C. Cummings III MD

The Cleveland Clinic, Cleveland, USA

E-mail: cummink2@ccf.org

\section{References}

1 Mertes PM, Laxenaire MC, Lienhard A, et al. Reducing the risk of anaphylaxis during anesthesia: guidelines for clinical practice. J Investig Allergol Clin Immunol 2005; 15: 91-101.

2 Dhonneur G, Combes X, Chassard D, Merle JC. Skin sensitivity to rocuronium and vecuronium: a randomized controlled prick-testing study in healthy volunteers. Anesth Analg 2004; 98: 986-9.

3 Kontou-Fili $K$. Patients with negative skin tests. Curr Opin Allergy Clin Immunol 2002; 2: 353-7.

4 Fisher MM, Merefield D, Baldo B. Failure to prevent an anaphylactic reaction to a second neuromuscular blocking drug during anaesthesia. Br J Anaesth 1999; 82: 770-3.

\section{Evaluating effectiveness of medical emergency teams}

To the Editor:

Brindley et al. ${ }^{1}$ are to be congratulated on their balanced appraisal of the MERIT study report. ${ }^{2}$ Unlike other commentators ${ }^{3,4}$ who have used its results to question the value of Medical Emergency Teams (MET), they rightly draw attention to the limitations of the study, which make it difficult to draw firm conclusions from its results. MERIT failed to find a clear advantage to the introduction of MET in a cluster randomized prospective trial, but the duration of the study period was short (only six months), and there was possibly cross-contamination between the study groups. Such teams are complex, introduction is labour-intensive and may be difficult, and after only 